\title{
Methods for reducing vibrations generated by shock equipment
}

\author{
Sergei Pushenko ${ }^{1}$, Evgeny Bozhko ${ }^{1}$, Irina Zolotuhina, ${ }^{1, *}$ \\ ${ }^{1}$ Don State Technical University, pl. Gagarina, 1, 344002, Rostov-on-Don, Russia
}

\begin{abstract}
Noise is one of the most unfavorable environmental factors in production facilities, for which it is most difficult to achieve compliance with sanitary standards. Prolonged exposure to intense vibrations and noise leads to noise and vibration diseases. The effect of noise on the human body is not limited to exposure to the organ of hearing. Irritation by noise is transmitted to the central and autonomic nervous systems, through them acts on the internal organs, leading to significant changes in the functional state of the organism. With pulsed and irregular noise, the degree of impact of noise on the body of workers increases. Most types of forging equipment relate to impact machines, during the operation of which impulse noise occurs, the levels of which at workplaces, in most cases, exceed the maximum permissible values and relate to those factors, the significance of which in some cases is greater than dustiness, high temperature, manipulation of heated workpieces. Therefore, the problem of reducing vibration and noise in the forge shops is extremely relevant and has great scientific and socio-economic significance. To solve the problem of noise reduction in forge shops, various options for reducing the noise activity of equipment have been developed/ It is possible to most effectively reduce noise by changing the material and shapes of the beds, noise-attenuating casings, oil baths for gears.
\end{abstract}

\section{Introduction}

All these measures on existing and running equipment are implemented through the use of passive noise protection measures, which in the current production are little feasible, and in most cases impossible at all. The problem of reducing noise in the source of its occurrence and bringing the noise levels to the maximum permissible values should be solved at the design stage. Such an approach to solving the problem of noise reduction is the most justified both from the scientific, technical and economic points of view. Work in this direction is carried out in far abroad countries and is almost completely absent in our country. This is explained, first of all, by the absence of reliable engineering methods for calculating noise and vibration, which would take into account the design features of forging machines and technological operating modes, made it possible to assess the expected noise levels at the stage of calculation and design and choose engineering

\footnotetext{
*Corresponding author: 5976765@mail.ru
} 
solutions to reduce noise levels up to standard values for each specific type of forging equipment.

\section{Methods}

When working in conditions of vibration, labor productivity decreases, the number of injuries increases. At some workplaces in agricultural production, vibrations exceed normalized values, and in some cases they are close to limit. The vibration levels on the controls do not always comply with the standards. Typically, low-frequency vibrations that negatively affect the body predominate in the vibration spectrum. Some types of vibration adversely affect the nervous and cardiovascular systems, the vestibular apparatus. The most harmful effect on the human body is provided by vibration, the frequency of which coincides with the frequency of natural vibrations of individual organs, the approximate values of which are as follows $(\mathrm{Hz})$ : stomach - $2 \ldots 3$; kidneys - $6 \ldots 8$; heart - 4 ... 6; intestines $-2 \ldots 4$; vestibular apparatus - 0.5..L, 3; eyes - $40 \ldots 100$, etc.

The effect on muscular reflexes reaches $20 \mathrm{~Hz}$; the tractor-loaded seat on the tractor has its own vibration frequency of $1.5 \ldots 1.8 \mathrm{~Hz}$, and the rear wheels of the tractor - $4 \mathrm{~Hz}$. The vibration is transmitted to the human body at the moment of contact with the vibrating object: when acting on the limbs, local vibration occurs, and on the whole body - general. Local vibration affects neuromuscular tissues and the musculoskeletal system and leads to spasms of peripheral vessels. With prolonged and intense vibrations, in some cases, a professional pathology develops (local vibration often leads to it): peripheral, cerebral or cerebral-peripheral vibrational disease. In the latter case, there are changes in cardiac activity, general excitement, or, conversely, inhibition, fatigue, the appearance of pain, a feeling of shaking of the internal organs, nausea. In these cases, vibrations also affect the osteoarticular apparatus, muscles, peripheral circulation, vision, hearing $[1,3,5,7,9]$. Local vibrations cause spasms of blood vessels that develop from the terminal phalanges of the fingers, spreading to the entire hand, forearm, and cover the vessels of the heart.

Workers can be exposed to occupational vibration through the use of power or pneumatic hand tools or other machinery, or by driving large transportation, construction or agricultural vehicles. Vibration that is generated through the use of powered hand tools, and is transmitted from the tool to the hand-arm system is referred to as hand-transmitted vibration. However, recent studies have also demonstrated that vibration can be transmitted through platforms workers are standing on, and in these situations, the point of contact is the feet. Workers can also be exposed to whole body vibration (WBV). WBV exposure occurs in occupations where workers are driving trucks, large earth moving vehicles, or where they are using hand tools where the amplitude of the vibration is great enough to be transmitted to other portions of the body, such as in workers using jack-hammers.

Exposure to WBV is of concern within the workforce because it's associated with the development of a number of negative health consequences including back and neck pain, and potentially, cardiovascular disease, the development of various neuropathies, digestive problems, headaches, dizziness, motion sickness and possibly cancer. However, workers exposed to WBV are often also exposed to a number of other risk factors that may contribute to the development of these negative health effects. These risk factors include maintaining a static posture for a long-period of time, torque or twisting of the abdomen to view the area around the vehicle, and heavy lifting that often occurs when a vehicle is being loaded or unloaded. In addition to vibration and the physical exposures associated with a job, there may be other co-exposures to chemicals or certain environmental conditions that contribute to the development of disease or injury in workers. Because most workers are exposed to multiple factors that may induced injury or illness, it's difficult to determine which factors pose the greatest risk for inducing injury or illness. Experimental studies, 
examining the effects of each of these factor individually on health outcomes can provide additional information that will help determine the contribution of each exposure factor to various health problems.

This review will describe the industrial sectors where vibration exposure is most prevalent and the health effects associated with exposure to HTV and WBV. Experimental methods used to measure and characterize vibration generated in various occupational settings are discussed, and models that have been used to uncover the etiology of vibrationinduced injuries. Although numerous studies have been published on both HTV and HBV, there are new epidemiological studies showing an increased risk of specific cancers with exposure to WBV. Therefore additional etiological studies need to be performed. New avenues for research are discussed below. Among the studies devoted to the study of vibrations and noise of forging equipment, the main achievements were obtained in the field of vibration reduction. The results of theoretical and experimental studies on reducing vibration of the equipment itself, protecting personnel and vibration-sensitive equipment are presented $[1,2,3,5]$.

The method of under-vibration isolation is widely and successfully applied, when the vibration activity of the hammer foundation is reduced by installing shock absorbers directly under the hammer's hammer. Rubber-metal elements, springs, pneumatic and other devices serve as a shock absorber for such structures. The disadvantages of this method include the high cost, significant dimensions, especially in the depth of laying, the complexity of installation. More technologically advanced is the scheme where rubberspring shock absorbers are used, made of thick-walled rubber pipes and cylindrical coil springs installed at the end.

A known implementation of vibration isolation of hammers when they are installed together on a reinforced concrete floating plate, which is mounted on 48 pneumatic shock absorbers.

The results of laboratory studies of viscous dampers are presented. These studies were carried out for the calculation of damping in the design of vibration isolation of the foundation. When taking into account the resistance forces, which are proportional to the speed of movement, the movement of the insulated installation after impact is described by the equation

$$
\left(m_{1}+m_{2}\right) * y^{\prime \prime}+\beta^{*} y+c y=0
$$

$\mathrm{m}_{1}$ - mass of falling woman;

$\mathrm{m}_{2}$ - the mass of the under-mass array, the sabot, the bed of the hammer;

$\beta$ - damping resistance in $\mathrm{kg} \mathrm{s} / \mathrm{cm}$;

$\mathrm{c}$ - stiffness coefficient, $\mathrm{kg} / \mathrm{cm}$.

The solution to this equation is obtained for the maximum amplitude of the bias. An analysis of this equation made it possible to obtain some dependences for determining a viscous fluid of a certain composition and to establish the sizes of dampers consisting of horizontal and vertical plates.

The equation describes the motion of a single-mass system and does not allow calculating the vibration spectrum of the foundation. In principle, it is possible to use plate dampers for vibration reduction purposes, which consist of a set of corrugated metal plates with gaskets. In some cases, it is possible to use oak pillows.

Protection from vibration of maintenance personnel and vibration-sensitive equipment is most effectively currently solved by methods of vibration isolation from vibration sources. The range of vibration waves propagating through the ground is limited by the frequency range of 8-30 Hz. Therefore, vibration isolation devices provide frequencies less, namely no more than $10-15 \mathrm{~Hz}$. 
At the present time, extensive experience has been gained with installation on shock absorbers, the rigidity of which is calculated on the basis of the task of choosing their optimal parameters $[4,6,7,8]$.

Currently available developments to reduce vibration are quite universal in nature for shock equipment and can be used for the equipment considered in this work. Most types of forge-and-press equipment belong to impact machines, during the operation of which impulse noise occurs. Noise levels at workplaces in most cases exceed permissible levels. The greatest noise level during hammer operation occurs in the processing zone. The sources of noise generation, duration, the level of noise created by each source are considered. According to the authors, the initial noise source on the stamping hammer is compressed air coming from the inter-stamping volume to reduce the distance between the stamp halves. The pressure and air temperature in the interstamp volume increases. An additional increase in pressure in the interstamping volume occurs due to gas generation during combustion of the stamp lubricant. Air exhaust from the inter-stamping volume creates a short-term impulse noise within $140 \mathrm{~dB}$, which leads to pain in the staff. The aim of this work is to further study the physical process of the occurrence of aerodynamic noise from an outgoing air stream and to find means of dealing with it, as well as the theoretical determination of the main structural dimensions of sound-damping devices depending on the final speed of the woman and the size of the stamp mirror.

When designing silencing devices, it is necessary to know the shape and dimensions of a free-flowing flat impulse jet propagating in a stationary medium, as well as the change in speed, pressure and temperature along its length and cross section. Experimental studies on a special installation simulating a flat channel with a variable output section area formed by two flat dies made it possible to establish a mixing zone with ambient air. It is this zone, according to the authors, that is the source of intense turbulent pulsations of the speed and pressure of the air stream, which propagate outside the stream in the form of sound vibrations, creating aerodynamic noise. As studies have shown, the initial section of the jet creates about $75 \%$ of the total sound power of the jet.

Therefore, the authors conclude that the reduction in aerodynamic noise during the work of the stamping hammer is associated with the destruction and braking of the jet of air flowing out of the interstamp volume when the stamps are closed, or the space around the stamp. Active sound-damping devices act on the air stream, preventing its speed from moving along the channel formed by the halves of the stamp as they close, changing the noise spectrum. Structurally, these devices are so profiled surface that at the moment of closing the halves of the stamp formed cavities or grooves that change the profile and the cross-sectional area of the channel. Air flow passing through such a channel, due to the presence of sudden expansions and turns, does not increase the speed above the values corresponding to the noise level $[9,10,11]$.

Passive sound-absorbing devices, without affecting the source of noise generation, limit the propagation of sound vibrations from it in the isolated region. Structurally, they are screens, grids installed around the dies at a certain angle to the plane of its connector and at a distance no greater than the length of the portion of the jet that generates the highest level of aerodynamic noise. The authors present the results of an approximate calculation of the volume of grooves and the height of overlap H] by the screen of the plane of the connector of the dies, the distance A from the side edge of the stamp and the angle of inclination a.

The height of the protrusion of the half of the stamp:

$$
\mathbf{A} \leq(\mathbf{5} \div \mathbf{6}) * \Delta \mathbf{H}
$$

Overlap safety factor $1,5-2$. 


$$
H_{1}=\Delta H^{*}\left(1+\quad A / \Delta H^{*} \operatorname{tg} a\right) * k
$$

The question of the appropriateness of using one or another constructive solution of a noise suppressing device should be decided for a particular stamp, its design, dimensions, strength characteristics and conditions of its operation. When measuring noise on a working rolling hammer, it was determined that the maximum noise value occurs before the stamps were closed during "hard" shocks, i.e. the speed of movement of the acoustic disturbance turns out to be greater than the speed of sound in the medium in which the acoustic wave propagates due to the impact of parts of the hammer, or the acoustic disturbance of the medium occurs before the shock. Therefore, the noise generation process of the stamping hammer was studied from the beginning of the movement of the upper stamp to the moment the stamps are closed. For a theoretical description, the interstamp volume is conventionally represented by a diffuser with a reduced rectangular cross-section. Dependencies are obtained for calculating the change in pressure and flow rate from the interstamping volume in time. The research results explain the occurrence of maximum aerodynamic noise during hard hitting, which results in a complete or almost complete closure of the stamp surfaces(Fig.1). On the first "soft" strokes, the peak value of the noise depends both on the parameters of the air jet flowing from the interstamp volume due to its reduction, and due to the distribution of forgings, which was studied in a laboratory setup [12].

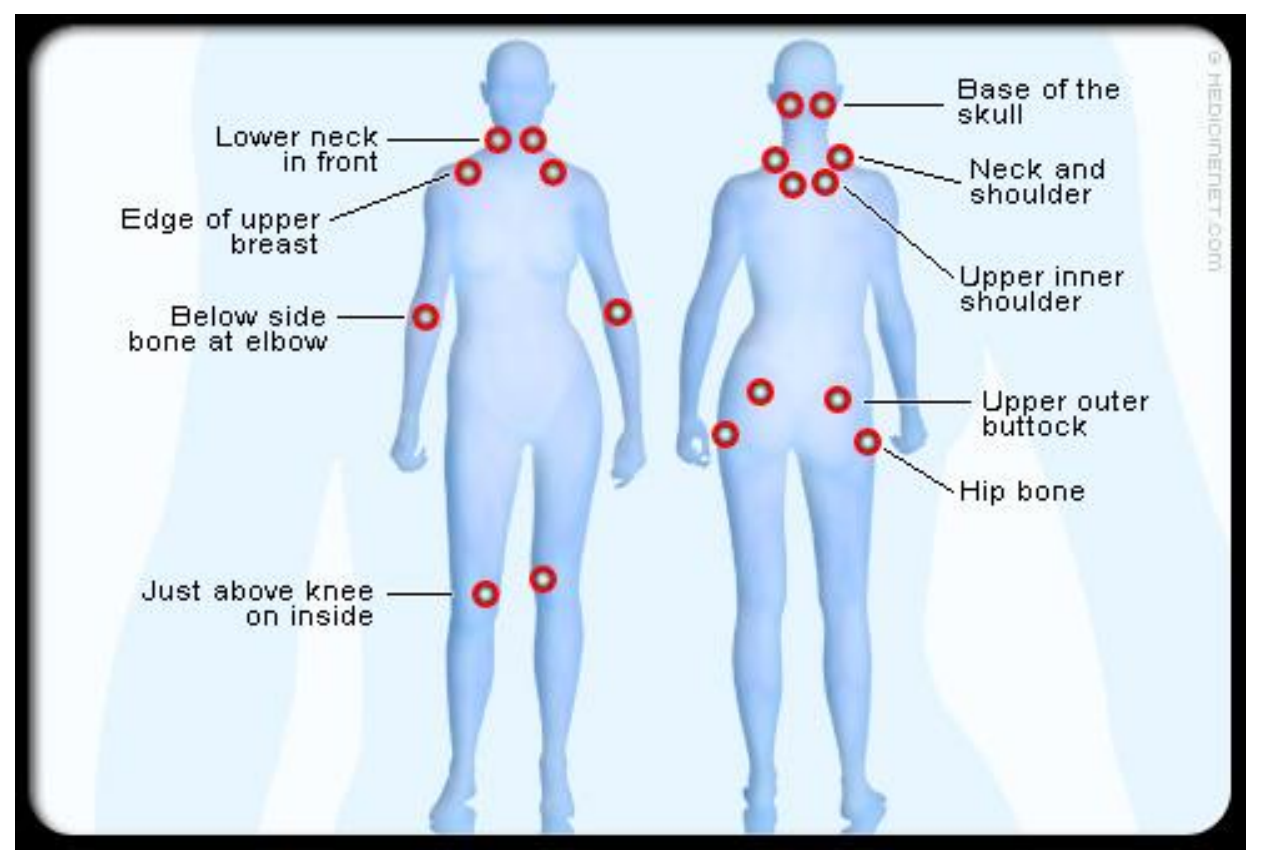

Fig. 1. Vibration influence points.

Based on experimental data and analysis of literary sources, the authors draw the following conclusions:

1. The stamping hammer is a source of two types of noise: aerodynamic from the air flowing out from the interstamp volume when the surfaces of the stamps approach the final hard blows and from the distribution of forgings, as well as the air stream flowing from the interstamp volume at the first soft strokes; mechanical, arising from fluctuations in the surfaces of the hammer caused by the impact of the stamp on the final "hard" strokes, or the stamp on forging on the first "soft" strokes and following the aerodynamic noise. 
2. The peak value of aerodynamic noise when working on a stamping hammer occurs due to a jet of air flowing out of the interstamp volume, or distribution of forgings and exceeds the subsequent mechanical noise by $15-30 \mathrm{~dB}$ depending on the speed of impact of the surfaces of the stamps and their sizes.

3. The sound power level of a subsonic jet with large Mach numbers and a sonic jet freely flowing out of the interstamp volume of the subsonic jet depends both on the speed of the jet and on its pressure on the edges of the dies when closed.

4. Measures to combat aerodynamic noise are qualitatively different from measures to combat mechanical noise and should be aimed at reducing the speed and movement of the air stream flowing from the interstamp volume to environmental pressure when the dies approach each other. Structural measures that can reduce aerodynamic noise should be aimed at changing the shape of the dies in order to reduce or prevent the generation of aerodynamic noise in the source.

\section{Results}

Intense noise emission is created by the frame and flywheel of a mechanical press. The vibrations of the bed and the flywheel are excited as a result of impacts in all movable joints of the press that occur at the moment of switching on and at the beginning of the movement of the crank or eccentric mechanism, that is, when the gaps in the joints of the connecting rod with the working shaft neck and slider are sampled bearings of the working shaft. The process of interaction between the stamp and the workpiece also has an impact character. The increase in stamping noise levels reaches $10 \mathrm{dBA}$. There is no noise of turning on the press during automatic operation. Noise levels do not change compared to the single start mode. Noise reduction can be achieved by ensuring a smooth operation of the switching process, which is realized by replacing mechanical cam couplings with friction or pneumatic ones. Noise during stamping is reduced when using beveled dies, instead of straight ones, which contributes to a smooth working process. These measures allow to reduce noise at the workplace of the puncher by $14 \mathrm{dBA}$. The use of such dies is justified when cutting parts of a large perimeter.

Preliminary operations (rolling, broaching, draft) of stamping on hammers are advisable to perform on separate equipment (forging rollers, hammers), since when performing preliminary and final stamping on a single hammer, strikes with different energies are applied, which requires a highly skilled blacksmith when controlling a hammer. The use of software control of the energy of the hammer strike eliminates the possibility of a collision of the mirror stamps during the preliminary operations.

Studies have established that stamp vibrations are transmitted through the die holder and rammers to the racks and the hammer cylinder and, in addition, during eccentric impacts through the head and guides, also to the hammer racks.

To reduce the vibrational excitation of the hammer parts, between the shabot and the die holder, as well as between the wedge and the die holder, a $2 \mathrm{~mm}$ thick rubber-asbestos gasket is installed, withstanding pressure up to $200 \mathrm{kgf} / \mathrm{mm} 2$ and temperature up to $500^{\circ}$ $\mathrm{C}$, or made of rubber-like material of the FKS brand, placed in order to avoid extrusion from the joint into the recess on the supporting surface of the die holder. At the same time, the noise level is $4 \mathrm{dBA}$.

It is possible to prevent the propagation of vibration from the hammer's hammer to the bed by installing damping gaskets at the joints or by coating the contact surfaces with elastic films, as well as by installing more powerful polyurethane shock absorbers and by increasing the tightening forces of the joints, preventing them from opening and impacting the contact surfaces of the parts during stamping. 
Gaskets are plates made of hard rubber, rubber-fabric material, asbestos-rubber, perforated neoprene, hard cardboard, pagulan, volculon materials from 1 to $6 \mathrm{~mm}$ thick, placed at the joints of the bed stands with the scabbard, and the racks with a sub-cylinder a stove and a cylinder of hammers and between wedges and racks. Elastic films at the same joints are made by applying gumming nairite composition or SKU-PFL polyurethane hammer in several layers with a total thickness of $1 \mathrm{~mm}$ to the primed contact surfaces of the parts. Measures to separate the butt surfaces and tighten the joints, together with the connection of the uprights in the upper section with tie bolts with an elastic element, reduce noise by 4-10 dBA. Rigid wedge fastening of the sub-cylinder plate to the uprights by the connection "lasochtokin tail" is also effective.

\section{Discussion}

The noise level on a hammer with a separate bed with an eccentric shock is 10$\urcorner-15 \mathrm{dBA}$ higher than with a central one, which is associated with the excitation of vibration of the racks by the beam through the guides. To exclude eccentric impacts, preliminary stamping operations must be performed on special equipment (for example, forging rollers), and gaskets made of pagulan type material with a thickness of $1 \mathrm{~mm}$, designed for pressure up to 10 , should be installed in the joints of guides with racks of the bed of the hammer. kgf / $\mathrm{mm} 2$ and temperature up to $80 \mathrm{C}$.

It is shown that the noise level from a hammer with a round bed profile and a structural damper built into the cavity of the bed stands is $10 \mathrm{dBA}$ lower than from a hammer with an open bed profile. The profile of the Shabot must be convex in order to avoid the formation of a reflected wave.

The structural damping of the bed can be increased by closing the rack profile by attaching screens with a sound-absorbing coating (for example, from a layer of mineral wool) on it and installing partitions inside the closed cavities with filling the cells between the partitions with sand, gravel or shot.

\section{Acknowledgements}

The study had no sponsorship.

\section{References}

1. A.T. Rybak, V.P. Zharov, A.V. Serdyukov, Russian Engineering Research 29(2), 194197 (2009)

2. A. Rybak, O. Lyakhnitskaya, MATEC Web of Conferences 132, 01001 (2017)

3. A.T. Rybak, A.R. Temirkanov, O.V. Lyakhnitskaya, Russian Engineering Research 38(9), 702-704 (2018)

4. A.T. Rybak, A.R. Temirkanov, O.V. Lyakhnitskaya, Russian Engineering Research 38(3), 212-217 (2018)

5. K. Kobzev, S. Shamshura, A. Chukarin, V. Bogdanovich, V. Kasyanov, MATEC Web of Conferences 226, 01022 (2018)

6. K. Kobzev, S. Shamshura, A. Chukarin, A. Buryanovand, V. Kasyanov, MATEC Web of Conferences 226, 01023 (2018)

7. K. Kobzev, A. Chukarin, IOP Conference Series: Earth and Environmental Science 403, 012145 (2019) 
8. K.O. Kobzev, E.S. Bozhko, A.V. Mozgovoi, M.D. Molev, N.I. Stuzhenko, IOP Conference Series: Materials Science and Engineering 680, 012014 (2019)

9. K.O. Kobzev, E.S. Bozhko, A.V. Mozgovoi, E.I. Kostromina, L.G. Babenko, IOP Conference Series: Materials Science and Engineering 680, 012013 (2019)

10. N. Kobzeva, V. Terentev, I. Zolotuhina, IOP Conference Series: Materials Science and Engineering 680, 012046 (2019)

11. N. Kobzeva, V. Terentev, I. Zolotuhina, IOP Conference Series: Materials Science and Engineering 680, 012011 (2019)

12. D. Larin, E. Staseva, E. Khetsuriani, IOP Conference Series: Materials Science and Engineering, Collection of materials of the XV International Scientific - Technical Conference Don State Technical University, 012016 (2019) 